\title{
Cross-Layer Adaptive Design for the Frame Length of IEEE 802.11 Networks
}

\author{
Feng Zheng and John Nelson \\ Department of Electronic and Computer Engineering \\ University of Limerick, Limerick, Ireland \\ Email: feng.zheng@ul.ie, john.nelson@ul.ie
}

\begin{abstract}
In this paper, we study the cross-layer (between MAC and PHY) design problem for IEEE 802.11 wireless networks. It is focused on the design of the optimal length of the frame body. The following results are obtained: 1) The optimal length of the frame body in logarithmic scale, expressed as $\log L_{f, o p t}$, can be coarsely approximated by a linear function of the signal-to-noise power ratio (SNR) $E_{b} / N_{0}$ (in $\mathbf{d B}$ ) and finely approximated by a second-order polynomial of $E_{b} / N_{0} ; 2$ ) The coefficients of the aforementioned approximation functions depend only on the data transmission rate and other parameters specified in the protocol, and they do not depend on the access mechanisms; 3) The number of active nodes has little effect on $L_{f, o p t}$, especially in the range of both low and high SNR; 4) The system throughput is sensitive to the length of the frame body $L_{f}$ when the SNR is low, while it is insensitive to $L_{f}$ in a considerably large neighborhood of $L_{f, o p t}$ when the SNR is high; and 5) Fragmentation can always increase the system throughput, but the increasing rate is conspicuous only in the low SNR regime.
\end{abstract}

\section{INTRODUCTION}

The IEEE 802.11 WLANs are being deployed widely and rapidly in many different environments. In the standards of IEEE 802.11 wireless networks, there are many optional design factors or parameters such as modulation schemes and transmission rates in the PHY layer, maximal number of retransmission tries, initial contention window size, and packet length in the MAC layer. While each layer's flexibility or adaptiveness to versatile products may be of primary concern in the protocols of wired networks, the integrated design of the whole systems across different layers to achieve optimal performance in some sense becomes important for wireless networks. Therefore, many research efforts have been made in cross-layer design, see, e.g., [5], [9], [12], [13], [15]. In [5], [12], the radio channel information is used to choose the modulation schemes and transmission rates to achieve good throughput. In [15], the packet error rate in Ricean fading channel is analyzed, and based on this result, an analytical model to evaluate the performance of IEEE 802.11 DCF in Ricean fading channel is given. In [13], the predictability of slow Rayleigh-fading channels is exploited to improve the network performance. In [9], a novel scheme for improving the network performance is investigated considering the physical layer's ability to decode simultaneously more

This work was supported by National Communications Network Research Centre, a Science Foundation Ireland Research Project, under Grant 03/IN3/1396. than one packet from multiple users. In [2], the authors investigated whether current IEEE 802.11 devices are able to comply with cross-layer and topology control requirements. The results reveal that many novel power control solutions cannot be efficiently implemented over existing IEEE 802.11 cards. Therefore, some simple and efficient cross-layer design approaches should be further studied.

The purpose of this study is to find the optimal packet length for the real time channel conditions. The basic idea is: if the packet length is too large, the packet retransmission rate will be high due to packet error; on the other hand, if the packet length is too small, much power will be wasted on the transmission of packet headers. Therefore some optimal packet length exists to achieve maximal throughput. The main reason for us to choose to design optimal packet length is that it can be easily adjusted in the MAC layer, and there are more flexibilities for such an adjustment, compared to the case of changing the transmission rate.

Notation: Throughout the paper, the energy per bit and per symbol for the PHY transmission is denoted as $E_{b}$ and $E_{s}$, respectively, the receiver noise is always assumed to be AWGN with (double sided) power spectral density being $N_{0} / 2$.

\section{Data Frame Structure And Modulation}

With the IEEE 802.11 MAC, any uncorrectable erroneous frame will be retransmitted by the sender up to a certain time [10]. Therefore the frame structure of the MAC and PHY layers is first described in the following.

\section{A. MAC Frame Structure}

The frame structure of the MAC protocol data units (MPDUs) is illustrated in Table I [1, p.34]. The Frame Body is a variable length field that contains information specific to individual frame types and subtypes. The minimum frame body is 0 octets. The maximum length frame body is defined in the control information bits. The FCS (frame check sequence) field is a 32-bit field containing an IEEE 32-bit cyclic redundancy code (CRC).

\begin{tabular}{|c|c|c|}
\hline MAC Header & Frame Body & FCS \\
\hline 30 octets & $0 \sim 2312$ octets & 4 octets \\
& TABLE I \\
MPDU FRAME FORMAT
\end{tabular}

In some cases (with a unicast receiver address), the MAC service data unit (MSDU) will be partitioned into MPDUs. 
This process is called fragmentation. The frame structure of the MSDU is depicted in [1, p.71].

The MPDUs resulting from the fragmentation of an MSDU are sent as independent transmissions, each of which is separately acknowledged. This permits transmission retries to occur per fragment, rather than per MSDU [1, pp.71-72].

\section{B. PHY Frame Structure for Frequency-Hopping Spread Spec- trum (FHSS) Systems}

In IEEE 802.11 wireless LAN, the MAC sublayer protocol data units (MPDUs) are mapped into a specific framing format suitable for transmission and receiving, as illustrated in Table II for the FHSS system, by the physical layer convergence procedure (PLCP) [1, p.152]. The header error check (HEC) field is a 16-bit CCITT CRC-16 error detection field.

\begin{tabular}{|c|c|c|c|c|c|}
\hline \multicolumn{2}{|c|}{ PLCP Preamble } & \multicolumn{3}{|c|}{ PLCP Header } & whitened PSDU \\
\hline SYNC & SFD & PLW & PSF & HEC & (MSDU) \\
\hline 80 bits & 16 bits & 12 bits & 4 bits & 16 bits \\
\multicolumn{6}{c}{ TABLE II } \\
PLCP FRAME FORMAT FOR FHSS SYSTEMS
\end{tabular}

C. PHY Frame Structure for Direct Sequence Spread Spectrum (DSSS) Systems

PHY frame structure for DSSS systems is illustrated in Table III [1, p.196]. The PLCP Header field is protected by the CCITT CRC-16 frame check sequence.

\begin{tabular}{|c|c|c|c|c|c|c|}
\hline \multicolumn{2}{|c|}{ PLCP Preamble } & \multicolumn{3}{c|}{ PLCP Header (48 bits) } & \multirow{2}{*}{ MPDU } \\
\hline SYNC & SFD & SIGNAL & SERVICE & LENGTH & CRC & \\
\hline 128 bits & 16 bits & 8 bits & 8 bits & 16 bits & 16 bits \\
\multicolumn{6}{|c}{ TABLE III } \\
\end{tabular}

\section{Data Modulation and Transmission}

In both FHSS and DSSS systems of 802.11 networks, the PLCP Preamble and Header fields are transmitted at $1 \mathrm{Mbits} / \mathrm{s}$, while the MSDU or MPDU may be transmitted at other rates specified by the PLCP Header field.

In FHSS 1 Mbits/s systems, the modulation scheme is twolevel Gaussian frequency shift keying (2GFSK). In FHSS 2 Mbits/s systems, the modulation scheme is four-level Gaussian frequency shift keying (4GFSK), where an incoming bit stream will be converted to 2-bit words or symbols.

In DSSS systems, the baseband modulations are differential binary phase shift keying (DBPSK) and differential quadrature phase shift keying (DQPSK) for the $1 \mathrm{Mbit} / \mathrm{s}$ and $2 \mathrm{Mbit} / \mathrm{s}$ data rates, respectively.

\section{Data Re-Transmission Rate}

In IEEE 802.11 wireless networks, data re-transmission is caused by two kinds of events. The first is data errors caused by fading and noise channels in the PHY layer, and the second is the data transmission collision caused by the competition for channel use in the MAC layer. In the event of data transmission collision, all the data contained in the collided MSDU or PPDU will be re-transmitted. In the event of data errors caused by fading and noise channels, the re-transmission can be categorized into two cases for FHSS systems, i.e., all the MSDU will be re-transmitted if the errors (uncorrectable) happen in the PLCP Header field; and only the erroneous MPDUs will be re-transmitted if the errors (uncorrectable) happen in the PLCP MSDU field. In the following, we will analyze data re-transmission rates for different cases.

\section{A. Re-Transmission Rate Caused by the PHY-layer Channel}

1) FHSS systems: Assume that the optimal receiver is used. To simplify the analysis, the channel fading is not considered. So the decision variable at the receiver is only subjected to the disturbance of the additive white Gaussian noise (AWGN). The probability of bit error, denoted as $q_{F H 1}$, for 1 Mbits/s FHSS systems can be expressed as [11, p.398]

$$
q_{F H 1}=Q\left(\sqrt{\alpha E_{b} / N_{0}}\right),
$$

where $Q(\cdot)$ denotes the $Q$-function and $\alpha$ is a constant whose value depends on the time-bandwidth product. For a typical case, this product is 0.3 , and then one has $\alpha=1.8$. We will assume that $\alpha$ takes this value in the subsequent analysis of this paper.

For 2 Mbits/s FHSS systems, it uses the 4GFSK modulation. Since an exact formula for the probability of symbol error is difficult to obtain for an $M$-ary FSK system, we use the upper bound to approximate it. From [11, p.401], the probability of symbol error is upper bounded by

$$
q_{s, F H 2} \leq 3 Q\left(\sqrt{\alpha E_{s} / N_{0}}\right) .
$$

Note that the above approximation becomes tight as $q_{s, F H 2} \leq$ $10^{-3}$. If Gray coding is used to map bits into symbols, the bit error probability can be approximated by [14, p.271]

$$
q_{F H 2} \approx q_{s, F H 2} / 2 \approx 3 Q\left(\sqrt{\alpha E_{s} / N_{0}}\right) / 2 .
$$

For an $(n, k)$ linear block code, suppose its minimum distance to be $d_{\min }$. Then the probability of an uncorrectable code word error, denoted as $P_{M}$, is upper bounded by [14, p.454]

$$
P_{M} \leq \sum_{m=\left\lfloor\frac{d_{m i n}-1}{2}\right\rfloor+1}^{n}\left(\begin{array}{c}
n \\
m
\end{array}\right) q^{m}(1-q)^{n-m},
$$

where $\lfloor\cdot\rfloor$ denotes the lower floor integer of its argument, and $q$ is the bit error probability. Since the exact formula for $P_{M}$ is difficult to obtain, we use (2) to approximate $P_{M}$.

For the PLCP Header field in FHSS systems, one has $n=32$ and $k=16$. From [8], one can see that $d_{\text {min }}=4$. Therefore, the probability of an uncorrectable PLCP Header error, denoted as $P_{H F H}$, is approximated by

$$
P_{H F H} \approx 1-\sum_{m=0}^{1}\left(\begin{array}{l}
32 \\
m
\end{array}\right) q_{F H 1}^{m}\left(1-q_{F H 1}\right)^{32-m} .
$$

For the MPDU field, one has $k=32$. Since the length of the frame body changes with applications, so does $n$. Suppose the length of the frame body is $L_{f}$ octets. Then we have $n=$ $8\left(L_{f}+34\right)$. From [7], we find that

$$
d_{\text {min }}= \begin{cases}6, & \text { if } 0 \leq L_{f} \leq 3 \\ 5, & \text { if } 4 \leq L_{f} \leq 341 \\ 4, & \text { if } 342 \leq L_{f} \leq 4095\end{cases}
$$


Therefore, the probability of an uncorrectable MPDU error, denoted as $P_{\mathrm{MPDU}}$, is approximated by

$P_{\mathrm{MPDU}} \approx\left\{\begin{array}{c}1-\sum_{m=0}^{2}\left(\begin{array}{l}\left({ }^{\left(L_{f}+34\right)}\right) \\ m\end{array}\right) q_{F H}^{m}\left(1-q_{F H}\right)^{8\left(L_{f}+34\right)-m} \\ \quad \text { if } 0 \leq L_{f} \leq 341 ; \\ 1-\sum_{m=0}^{1}\left({ }^{8\left(L_{f}+34\right)}{ }_{m}\right) q_{F H}^{m}\left(1-q_{F H}\right)^{8\left(L_{f}+34\right)-m} \\ \quad \text { if } 342 \leq L_{f} \leq 4095,\end{array}\right.$

where $q_{F H}$ will be substituted by, respectively, $q_{F H 1}$ for 1 Mbits/s FHSS systems and $q_{F H 2}$ for 2 Mbits/s FHSS systems.

2) DSSS systems: Here it is again assumed that the optimal receiver is used and the channel fading is not considered. The probability of bit error, denoted as $q_{D S 1}$, for $1 \mathrm{Mbits} / \mathrm{s}$ DSSS systems can be expressed as [14, p.274]

$$
q_{D S 1}=0.5 e^{-E_{b} / N_{0}} .
$$

For $2 \mathrm{Mbits} / \mathrm{s}$ DSSS systems, it uses the DQPSK modulation. Suppose the Gray coding is used for the source coding. Then the probability of bit error, denoted as $q_{D S 2}$, can be expressed as [14, p.275]

$$
q_{D S 2}=Q_{1}(a, b)-0.5 I_{0}(a b) e^{-a^{2}+b^{2} / 2} .
$$

where $Q_{1}$ and $I_{0}$ denote the Marcum $Q$-function and modified Bessel function, respectively, and the parameters $a$ and $b$ are defined as $a=\sqrt{(2-\sqrt{2}) E_{b} / N_{0}}, b=\sqrt{(2+\sqrt{2}) E_{b} / N_{0}}$.

For the PLCP Header field in DSSS systems, one has $n=48$ and $k=16$. From [8], one can see that $d_{\text {min }}=4$. Therefore, the probability of an uncorrectable PLCP Header error, denoted as $P_{H D S}$, is approximated by

$$
P_{H D S} \approx 1-\sum_{m=0}^{1}\left(\begin{array}{l}
48 \\
m
\end{array}\right) q_{D S 1}^{m}\left(1-q_{D S 1}\right)^{48-m} .
$$

To calculate the probability of an uncorrectable MPDU error in DSSS systems, we first revise equation (4) as follows:

$$
d_{\text {min }}= \begin{cases}6, & \text { if } 0 \leq L_{f} \leq 3 \\ 5, & \text { if } 4 \leq L_{f} \leq 341 \\ 4, & \text { if } 342 \leq L_{f} \leq 7966 \\ 3, & \text { if } 7967 \leq L_{f} \leq 8191\end{cases}
$$

Then it is easy to find (5) still applies to DSSS systems, i.e.,

$P_{\mathrm{MPDU}} \approx\left\{\begin{array}{c}1-\sum_{m=0}^{2}\left({ }^{8\left(L_{f}+34\right)}\right) q_{D S}^{m}\left(1-q_{D S}\right)^{8\left(L_{f}+34\right)-m} \\ \quad \text { if } 0 \leq L_{f} \leq 341 ; \\ 1-\sum_{m=0}^{1}\left({ }^{8\left(L_{f}+34\right)}\right) q_{D S}^{m}\left(1-q_{D S}\right)^{8\left(L_{f}+34\right)-m} \\ \quad \text { if } 342 \leq L_{f} \leq 8191,\end{array}\right.$

where $q_{D S}$ is substituted with $q_{D S 1}$ for 1 Mbits/s DSSS systems and $q_{D S 2}$ for 2 Mbits/s DSSS systems, respectively.

Finally, it is pointed out that we will assume that the control fields such as ACK, CTS, and RTS will be always correctly received by the destination. This is based on the following two reasons. First, the lengths of their bits contained in one frame are 112, 112, and 160 for ACK, CTS, and RTS respectively. The lengths are much shorter than that of an MPDU. Thus the bit errors, if any, in these control fields can be corrected by their FCS codes. Second, the transmission rate for these control fields may be lower than the rates used to transmit the data frames if multiple rates are used in the networks [1, p.73, p.95]. Therefore, the frame error probability of the control fields can be neglected compared to the frame error probability of MPDUs. For the frame structures of ACK, CTS, and RTS, readers are referred to [1, pp.41-42]

\section{B. Re-Transmission Rate Caused by the MAC-layer Channel}

The IEEE 802.11 uses a CSMA/CA MAC protocol with binary exponential backoff, referred to as the distributed coordination function (DCF), to access the medium. Two medium access techniques are used in DCF: the basic access mechanism and the four-way handshaking RTS/CTS mechanism, which are described in [1], [3]. Under these access mechanisms, a station may experience several collisions before it successfully gets the radio channel to transmit data. Suppose there are $N$ contending stations in the concerned network. Let $\tau$ denote the probability that a station transmits in a randomly chosen time slot, $W_{0}$ the initial contention window size, and $m$ the maximal number of re-transmission tries. Then $\tau$ can be found by solving the following equations [3]:

$$
\begin{aligned}
\tau & =\frac{2(1-2 p)}{(1-2 p)\left(W_{0}+1\right)+p W_{0}\left(1-(2 p)^{m}\right)}, \\
p & =1-(1-\tau)^{N-1} .
\end{aligned}
$$

Let $P_{C}$ denote the probability that the transmissions of the $N$ stations are colliding, and $P_{S}$ the probability that a transmission of one of the $N$ stations is successful in the sense of the MAC layer, i.e., one station has captured the MAC channel without colliding with other stations. Then we have [6]

$$
\begin{aligned}
P_{S} & =\frac{N \tau(1-\tau)^{N-1}}{1-(1-\tau)^{N}}, \\
P_{C} & =\frac{1-(1-\tau)^{N}-N \tau(1-\tau)^{N-1}}{1-(1-\tau)^{N}} .
\end{aligned}
$$

\section{EfFective Throughput of the 802.11 Networks}

\section{A. The Case of No Fragmentation}

In the case of no fragmentation, only one MPDU is contained in one PPDU. Let us introduce the concepts of an epoch and a virtual transmission time, with the latter being similar to the one introduced in [6]. An epoch is the time interval between two successful transmissions of one MPDU for a station. A virtual transmission time is the time interval between two successful captures of the the MAC layer channel for a station. Therefore one virtual transmission time includes one successful transmission try of data and maybe several collisions with the transmission tries of other stations, while one epoch includes one successful transmission of data and maybe several virtual transmissions, as illustrated in Fig 1.

Define $N_{\mathrm{idl}}$ to be the number of consecutive idle slots and $N_{\text {col }}$ to be the number of collisions in one virtual transmission 


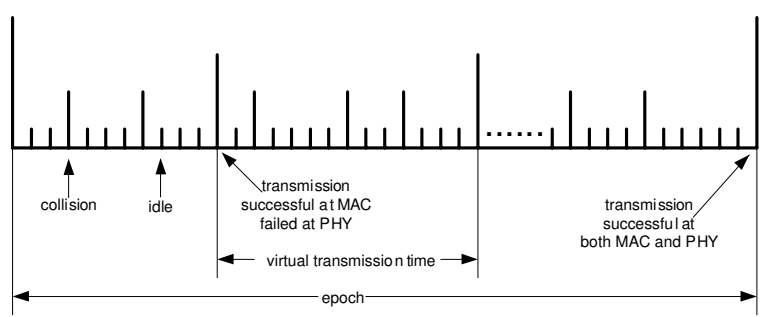

Fig. 1. Epoch structure for the data transmission in 802.11 networks considering both MAC collision and PHY failure.

time. Let $N_{\text {vir }}$ be the number of the virtual transmissions in one epoch. Consider one virtual transmission. Let $t=1$ denote the starting time (in slot) after a collision or the immediately former virtual transmission. Note that the probability that a station will transmit data at a given time slot is $\tau$. By the argument similar to [6], we can obtain

$$
\begin{aligned}
\mathcal{E}\left(N_{\mathrm{idl}}\right) & =\frac{(1-\tau)^{N}}{1-(1-\tau)^{N}} \\
\mathcal{E}\left(N_{\mathrm{col}}\right) & =\frac{1-(1-\tau)^{N}-N \tau(1-\tau)^{N-1}}{N \tau(1-\tau)^{N-1}} .
\end{aligned}
$$

Note that for an MPDU to be successfully received, both the PLCP header field and FCS check field should be correct. Suppose that the errors of PLCP and FCS happen independently. Denote by $P_{\text {suc }}$ the probability that an MPDU has been successfully received given that it is transmitted. Then

$$
P_{\mathrm{suc}}=\left(1-P_{\mathrm{Hdr}}\right)\left(1-P_{\mathrm{MPDU}}\right),
$$

where $P_{\mathrm{Hdr}}$ denotes the probability of detectable MAC header errors given that the header is transmitted. Therefore one has $\operatorname{Pr}\left\{N_{\text {vir }}=k\right\}=\operatorname{Pr}\{\{$ all previous $k-1$ data transmissions $\}$ are failed $\bigcap\{$ the $k$-th data transmission is successful $\}\}$

$$
=\left(1-P_{\text {suc }}\right)^{k-1} P_{\text {suc }} \text {, }
$$

$\mathcal{E}\left(N_{\text {vir }}\right)=\sum_{k=1}^{\infty} k\left(1-P_{\text {suc }}\right)^{k-1} P_{\text {suc }}=\frac{1}{P_{\text {suc }}}$.

Let $\sigma$ be the duration of an empty slot time, $T_{s}$ the average time the channel is sensed busy because of a data transmission attempt, $T_{c}$ the average time the channel is sensed busy by each station during a collision, and $T_{\mathrm{MPDU}}$ the time needed to transmit one MPDU:

$$
T_{\mathrm{MPDU}}=8\left(L_{f}+L_{\mathrm{MAC}_{\mathrm{hdr}}}+L_{\mathrm{FCS}}\right) / R=8\left(L_{f}+34\right) / R,
$$

where $R$ is the data transmission rate for the MPDU or MSDU, and $L_{\mathrm{MAC}_{\mathrm{hdr}}}$ and $L_{\mathrm{FCS}}$ denote the length of MAC header and FCS, respectively. Let $T_{f}$ be the time needed to transmit one frame body: $T_{f}=8 L_{f} / R$. The average time which the channel spends on colliding transmissions and idle slots in a virtual transmission time is

$$
T_{c \& i}=\xi T_{c}+(1-\tau) \sigma /(N \tau) .
$$

where $\xi=\frac{1-(1-\tau)^{N}}{N \tau(1-\tau)^{N-1}}-1$. Therefore in one epoch, the average transmission time will be given by $\mathcal{E}\left(N_{\text {vir }}\right)\left(T_{s}+T_{c \& i}\right)$. The throughput of the channel can be expressed as

$$
\rho_{1}=\frac{T_{f}}{\mathcal{E}\left(N_{\mathrm{vir}}\right)\left(T_{s}+T_{c \& i}\right)}=\frac{T_{f} P_{\mathrm{suc}}}{T_{s}+\xi T_{c}+(1-\tau) \sigma /(N \tau)} .
$$

The expressions of $T_{s}$ and $T_{c}$ for both basic and RTS/CTS accesses are given by (14) and (17), respectively, in [4].

\section{B. The Case of Fragmentation}

In this case, each MPDU in an MSDU will be acknowledged independently and the transmission retries will occur per fragment, rather than per MSDU. Suppose the error probability of all the MPDUs are independent of each other. Then all the MPDUs will experience the same average number of transmission retries to be correctly received. Therefore, following the same development as the preceding subsection, the throughput of the channel can now be expressed as

$$
\rho_{2}=\frac{T_{f} N_{\mathrm{MPDU}} P_{\mathrm{suc}}}{T_{s}+\xi T_{c}+(1-\tau) \sigma /(N \tau)},
$$

where $N_{\mathrm{MPDU}}$ is the number of MPDUs contained in one MSDU. $T_{s}$ and $T_{c}$ will be calculated in the following way. For basic access case, they are

$$
\left\{\begin{array}{l}
T_{s}^{\mathrm{bas}}=T_{\mathrm{PHY}}+N_{\mathrm{MPDU}} T_{\mathrm{MPDU}}+\mathrm{SIFS}+2 \delta+\mathrm{ACK}+\mathrm{DIFS}, \\
T_{c}^{\mathrm{bas}}=T_{\mathrm{PHY}}+N_{\mathrm{MPDU}} T_{\mathrm{MPDU}}+\mathrm{DIFS}+\delta,
\end{array}\right.
$$

For RTS/CTS access case, they are

$$
\left\{\begin{aligned}
T_{s}^{\mathrm{rts}}= & T_{\mathrm{PHY}}+N_{\mathrm{MPDU}} T_{\mathrm{MPDU}}+3 \cdot \mathrm{SIFS}+4 \delta+\mathrm{ACK} \\
& +\mathrm{DIFS}+\mathrm{RTS}+\mathrm{CTS} \\
T_{c}^{\mathrm{rts}}= & \mathrm{RTS}+\mathrm{DIFS}+\delta .
\end{aligned}\right.
$$

\section{Optimization Design}

In this section, we investigate how to choose the optimal MAC layer parameters. Since the length of the frame body and the number of MPDUs contained in one MSDU can be easily adjusted in applications, we will focus our attention on the optimization of throughput $\rho$ with respect to $L_{f}$ and $N_{\text {MPDU }}$.

\section{A. The Case of No Fragmentation}

For notational simplicity, let us define

$$
g\left(L_{f}\right)=T_{s}+\xi T_{c}+(1-\tau) \sigma /(N \tau) .
$$

Differentiating $\rho_{1}$ with respect to $L_{f}$ yields

$\frac{d \rho_{1}}{d L_{f}}=\frac{1}{g^{2}\left(L_{f}\right)}\left\{g\left(L_{f}\right) \frac{d\left(T_{f} P_{\mathrm{suc}}\right)}{d L_{f}}-T_{f} P_{\mathrm{suc}} \frac{d\left[T_{s}+\xi T_{c}\right]}{d L_{f}}\right\}$.

From equations (9), (11) and (12), we have

$$
\left\{\begin{array}{l}
\frac{d T_{f}}{d L_{f}}=\frac{8}{R}, \quad \frac{d T_{s}}{d L_{f}}=\frac{8}{R}, \\
\frac{d P_{\mathrm{suc}}}{d L_{f}}=-\left(1-P_{\mathrm{Hdr}}\right) \frac{d P_{\mathrm{MPDU}}}{d L_{f}}, \\
\frac{d T_{c}}{d L_{f}}=\left\{\begin{array}{cc}
\frac{8}{R}, & \text { for basic access; } \\
0, & \text { for RTS/CTS access. }
\end{array}\right.
\end{array}\right.
$$


From (5) or (7) we have

$$
\begin{aligned}
& h\left(L_{f}\right):=\frac{d P_{\mathrm{MPDU}}}{d L_{f}}= \\
& \left\{\begin{array}{c}
-8(1-q)^{\zeta} \ln (1-q)-8 q(1-q)^{\zeta-1}-8 \zeta q(1-q)^{\zeta-1} \ln (1-q) \\
-4(2 \zeta-1) q^{2}(1-q)^{\zeta-2}-4 \zeta[\zeta-1] q^{2}(1-q)^{\zeta-2} \ln (1-q), \\
\text { if } 0 \leq L_{f} \leq 341 ; \\
-8(1-q)^{\zeta} \ln (1-q)-8 q(1-q)^{\zeta-1}-8 \zeta q(1-q)^{\zeta-1} \ln (1-q), \\
\text { if } 342 \leq L_{f} \leq 8191,
\end{array}\right.
\end{aligned}
$$

where $\zeta=8\left(L_{f}+34\right), q$ takes values of $q_{F H}$ and $q_{D S}$ for FHSS and DSSS systems respectively. Substituting (14) into (13) yields

$\frac{d \rho_{1}}{d L_{f}}=\left\{\begin{array}{c}\frac{1}{g^{2}\left(L_{f}\right)}\left\{g\left(L_{f}\right)\left[\frac{8 P_{\mathrm{suc}}}{R}-T_{f}\left(1-P_{\mathrm{Hdr}}\right) h\left(L_{f}\right)\right]\right. \\ \left.-\frac{8 P_{\mathrm{suc}} T_{f}}{R}(1+\xi)\right\}, \text { for basic access mechanism; } \\ \frac{1}{g^{2}\left(L_{f}\right)}\left\{g\left(L_{f}\right)\left[\frac{8 P_{\mathrm{suc}}}{R}-T_{f}\left(1-P_{\mathrm{Hdr}}\right) h\left(L_{f}\right)\right]-\frac{8 P_{\mathrm{suc}} T_{f}}{R}\right\}, \\ \quad \text { for RTS/CTS access mechanism. }\end{array}\right.$

Therefore, the optimal $L_{f}$ can be obtained by solving the following equation:

$\left\{\begin{array}{l}g\left(L_{f}\right)\left(1-P_{\mathrm{MPDU}}\right)-L_{f} g\left(L_{f}\right) h\left(L_{f}\right)-\frac{8\left(1-P_{\mathrm{MPDU}}\right) L_{f}(1+\xi)}{R}=0, \\ \quad \text { for basic access mechanism; } \\ g\left(L_{f}\right)\left(1-P_{\mathrm{MPDU}}\right)-L_{f} g\left(L_{f}\right) h\left(L_{f}\right)-\frac{8\left(1-P_{\mathrm{MPDU}}\right) L_{f}}{R}=0, \\ \quad \text { for RTS/CTS access mechanism. }\end{array}\right.$

\section{B. The Case of Fragmentation}

Using the argument similar to the previous subsection, it can be proven that

$$
\frac{\partial \rho_{2}}{\partial N_{\mathrm{MPDU}}}>0
$$

for both basic access mechanism and RTS/CTS access mechanism. Therefore the throughput will increase monotonously with $N_{\text {MPDU }}$. The remaining objective is to find optimal $L_{f}$, which is the solution of the following equations.

$$
\begin{aligned}
& g\left(L_{f}\right)\left(1-P_{\mathrm{MPDU}}\right)-L_{f} g\left(L_{f}\right) h\left(L_{f}\right) \\
& \quad-8 N_{\mathrm{MPDU}}\left(1-P_{\mathrm{MPDU}}\right) L_{f}(1+\xi) / R=0, \text { for basic access; } \\
& g\left(L_{f}\right)\left(1-P_{\mathrm{MPDU}}\right)-L_{f} g\left(L_{f}\right) h\left(L_{f}\right) \\
& \quad-8 N_{\mathrm{MPDU}}\left(1-P_{\mathrm{MPDU}}\right) L_{f} / R=0, \text { for RTS/CTS access. }
\end{aligned}
$$

In summary, the optimal $L_{f}$ can be found by solving (15), (16), or (17) respectively for the corresponding case.

\section{NumERiCAL Results}

The parameters used in this section are taken from the standard IEEE 802.11 for the basic systems [1, p.34], which are listed in Table IV for easy citation.

\begin{tabular}{|c|c|c|c|c|c|c|c|}
\hline & $\sigma$ & SIFS & DIFS & $\delta$ & $W_{0}$ & $m$ & $L_{f, \max }$ \\
\hline FHSS & $50 \mu \mathrm{s}$ & $28 \mu \mathrm{s}$ & $128 \mu \mathrm{s}$ & $1 \mu \mathrm{s}$ & 16 & 6 & 4095 \\
\hline DSSS & $20 \mu \mathrm{s}$ & $10 \mu \mathrm{s}$ & $50 \mu \mathrm{s}$ & $1 \mu \mathrm{s}$ & 32 & 5 & 8191 \\
\hline
\end{tabular}

TABLE IV

PHY AND MAC PARAMETERS FOR BOTH FHSS AND DSSS SYSTEMS

In practical applications, the number of active nodes $(N)$ is subjected to variation and it is difficult to estimate this number.
So we first investigate how the optimal length of the frame body, denoted as $L_{f, o p t}$, varies with the number $N$. The results are illustrated in Fig. 2. From this figure, it can be observed that $L_{f, o p t}$ does not vary too much with $N$. Especially, in the range of both low and high $\operatorname{SNR}\left(E_{b} / N_{0}\right), L_{f, o p t}$ almost does not change with $N$. Similar results are obtained for other kinds of systems. Based upon this observation, we can fix $N$ to be some constant to investigate how $L_{f, o p t}$ depends on other parameters. In the following, we will fix $N=10$.

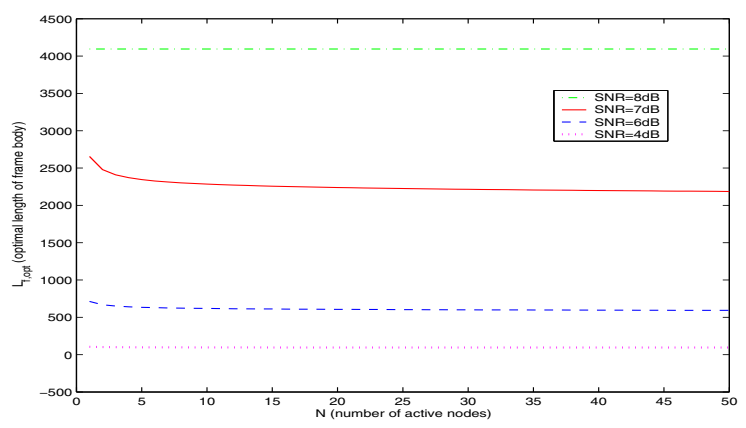

Fig. 2. The optimal length of the frame body varies with respect to the number of active nodes for the FHSS, 2Mbits/s, basic access system.

The relationship between $L_{f, o p t}$ and the SNR is illustrated in Fig. 3. From this figure it can be found that $\log _{10} L_{f, o p t}$ is well approximated by a linear function or a second-order polynomial of the SNR. This approximation function depends on the transmission rate, but it does not depend on the type of access mechanisms. Define a saturation function sat as follows

$$
\operatorname{sat}\left(f(x) ; x_{\max }\right)= \begin{cases}f(x) & \text { if } 0 \leq f(x) \leq x_{\max } \\ 0 & \text { if } f(x)<0 \\ x_{\max } & \text { if } f(x)>x_{\max }\end{cases}
$$

Then for the FHSS systems, we have

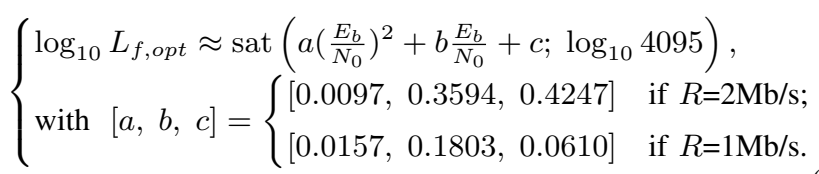

For the DSSS systems, we have

$\left\{\begin{array}{l}\log _{10} L_{f, o p t} \approx \operatorname{sat}\left(a\left(\frac{E_{b}}{N_{0}}\right)^{2}+b \frac{E_{b}}{N_{0}}+c ; \log _{10} 8191\right), \\ \text { with }[a, b, c]= \begin{cases}{[0.0174,0.0981,-0.1946]} & \text { if } R=2 \mathrm{Mb} / \mathrm{s} ; \\ {[0.0240,0.1089,-0.2463]} & \text { if } R=1 \mathrm{Mb} / \mathrm{s} .\end{cases} \end{array}\right.$

Next we investigate how sensitive the throughput is against the length of the frame body. This sensitivity is shown in Fig. 4. It is found that the system throughput is sensitive to $L_{f}$ when the SNR is low, while it is insensitive to $L_{f}$ for both FHSS and DSSS systems in a considerably large neighborhood of $L_{f, o p t}$ when the SNR is high. Fig. 4 only depicts the result for $2 \mathrm{Mb} / \mathrm{s}$ and basic access systems. For 1Mbits/s and RTS/CTS access systems, similar results are also obtained.

Finally, we investigate the effect of the fragmentation on the system performance. Due to space limit, the results are not shown here. The following conclusions are obtained: i) 

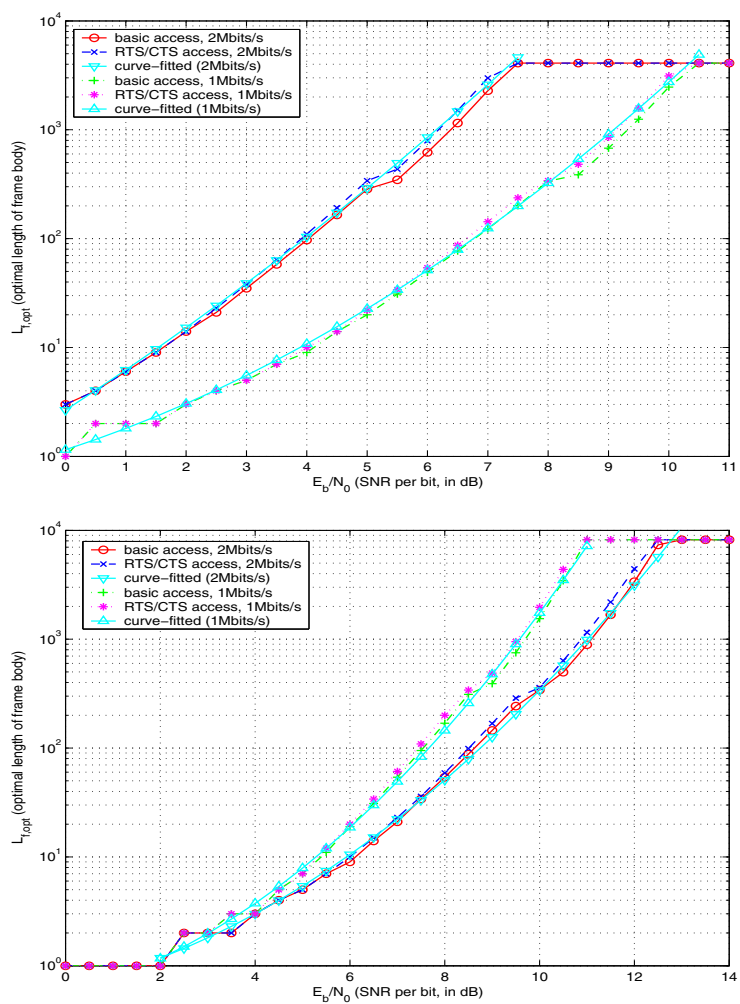

Fig. 3. The second order curve approximation for the relationship between $L_{f, o p t} \underset{0.18}{\operatorname{and}} E_{b} / N_{0}$. Upper: FHSS; Lower: DSSS.
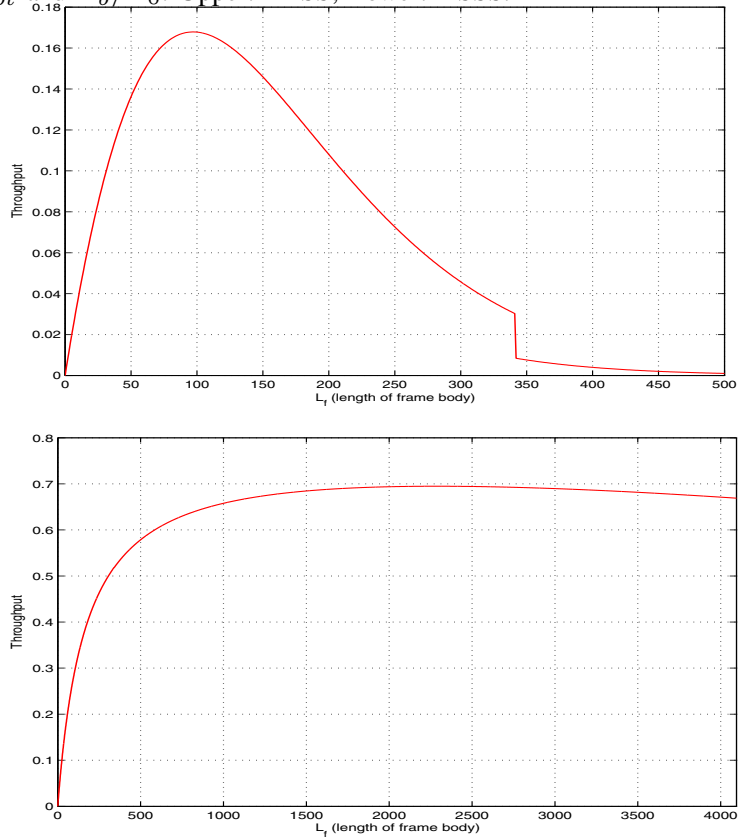

Fig. 4. The sensitivity of the throughput against the length of the frame body. All are for 2Mbits/s and basic access systems. Similar results are found for DSSS systems. Upper: FHSS, SNR $=4 \mathrm{~dB}, L_{f, o p t}=97$; Lower: FHSS, $\mathrm{SNR}=7 \mathrm{~dB}, L_{f, o p t}=2285$.

When the SNR is low, the system throughput increases with $N_{\text {MPDU }}$ and the increasing rate is considerably large, while the variation of the system throughput with respect to $N_{\text {MPDU }}$ can be hardly seen when the SNR is high, even though the system throughput indeed increases with $N_{\text {MPDU }}$ in this case; ii) $L_{f, o p t}$ decreases with $N_{\text {MPDU }}$ monotonously. Therefore, fragmentation is instrumental only in the low SNR regime as far as system throughput is concerned.

\section{CONCLUSIONS}

In this paper, we have investigated the cross-layer (between MAC and PHY) design problem for IEEE 802.11 wireless networks. It is found that

1) The optimal length of the frame body in logarithmic scale, expressed as $\log L_{f, o p t}$, can be coarsely approximated by a linear function of $E_{b} / N_{0}$ (in $\mathrm{dB}$ ) and finely approximated by a second-order polynomial of $E_{b} / N_{0}$ (in $\mathrm{dB}$ ). The coefficients of the approximation function depend only on the data transmission rate and other parameter specified in the protocol, and they do not depend on the access mechanisms;

2) The number of active nodes $N$ has little effect on $L_{f, o p t}$, especially in the range of both low and high SNR;

3) The system throughput is sensitive to $L_{f}$ when the SNR is low, while it is insensitive to $L_{f}$ in a considerably large neighborhood of $L_{f, o p t}$ when the SNR is high;

4) Fragmentation can always increase the system throughput, but the increasing rate is conspicuous only in the low SNR regime.

\section{REFERENCES}

[1] IEEE Standard for Wireless Lan Medium Access Control (MAC) and Physical Layer(PHY) Specification, 1999 Edition (R2003).

[2] F. B. Abdesslem and L. Iannone et al.. On the feasibility of power control in current ieee 802.11 devices. 4th IEEE Int. Workshops Pervasive Computing \& Commun., 13-17 March 2006.

[3] G. Bianchi. IEEE 802.11 - saturation throughput analysis. IEEE Commun. Lett., 2:318-320, 1998.

[4] G. Bianchi. Performance analysis of the IEEE 802.11 distributed coordination function. IEEE J. Sel. Areas Commun., 18:535-547, 2000.

[5] S. Bouam and J. B. Othman. A 802.11 multiservices cross-layer approach for QoS management. 2004 IEEE VTC-Fall, pp.2698-2702, 26-29 Sept. 2004.

[6] F. Calì, M. Conti, and E. Gregori. Dynamic tuning of the IEEE 802.11 protocol to achieve a theoretical throughput limit. IEEE/ACM Trans. Networking, 8:785-799, 2000.

[7] G. Castagnoli, S. Bräuer, and M. Herrmann. Optimization of cyclic redundancy-check codes with 24 and 32 parity bits. IEEE Trans. Commun., 41:883-892, 1993.

[8] G. Castagnoli, J. Ganz, and P. Graber. Optimum cyclic redundancycheck codes with 16-bit redundancy. IEEE Trans. Commun., 38:111$114,1990$.

[9] D. S. Chan and T. Berger. Performance and cross-layer design of CSMA for wireless networks with multipacket reception. 38th Asilomar Conf. Signals, Systems Computers, pp.1917-1921, 7-10 Nov. 2004.

[10] S. Choi, Y. Choi, and I. Lee. IEEE 802.11 MAC-level FEC scheme with retransmission combining. IEEE Trans. Wireless Commun., 5:203-211, 2006.

[11] S. Haykin. Communication Systems. John Wiley, 4th ed., 2001.

[12] W.-C. Liu, L.-C. Wang, and Y.-W. Lin. Physical layer effects on the MAC goodput performance for the rate adaptive IEEE 802.11a/g WLAN. In 2004 IEEE WCNC, pp.1873-1878, 21-25 March 2004.

[13] P. P. Pham, S. Perreau, and A. Jayasuriya. New cross-layer design approach to ad hoc networks under Rayleigh fading. IEEE J. Sel. Areas Commun., 23:28-39, 2005.

[14] J. G. Proakis. Digital Communications. McGraw-Hill, 4th ed., 2001.

[15] X. Zhou and J. Caffery. Cross-layer analysis of IEEE 802.11 DCF in burst-error fading channels with diversity. 2005 Int. Conf. Wireless Networks, Commun. Mobile Computing, pp.704-709, 13-16 June 2005. 